\title{
A Collective Motion Algorithm for Tracking Time-Dependent Boundaries
}

\author{
Ioana Triandaf and Ira B. Schwartz \\ Naval Research Laboratory, Code 6792, Plasma Physics Division, Washington, DC \\ 20375
}

\section{Introduction}

We propose and study a method for a multi-agent system of autonomous vehicles to perform the exploration of a non-stationary environment. The goal of this exploration is to reach a designated target geometry, typically a boundary, and to describe that boundary. The current paper relates to the general objective of studying motions of swarms, which we understand as a collection of autonomous entities which rely on local sensing and simple behavior, interacting in a way that a more complex behavior of the whole group emerges from local interactions [3]. This type of behavior is well-known in natural phenomena such as schools of fish [3] and colonies of insects [4]. One can find in the literature various other methods of simulating motions of distributed systems inspired by biology [4], [5], and by economics-based concepts [7]. The applications of such motions are quite broad since substituting agents for humans is desirable in many areas such as de-mining operations [6], mapping and exploration [8], navigation control [9], formation flying [10] and military planning [3].

The algorithm presented here models a group of agents to move on a given surface or within a prescribed volume, find its boundary and track it, while communicating with each other and spreading simultaneously over the surface. The environment in which 
the motion takes place varies in time. Such a method may provide insight to a better understanding of motions of swarms in biology [4]. The motion of swarms in military applications has been considered in recent years [3], since it offers an unmanned, dependable, flexible, self-organized type of network to achieve various military objectives, while replacing the burden of comprehensive communication, since the swarms interact primarily with their neighbors.

The particular goal of identifying and tracking the boundary of a surface which we address here, is analogous to the problem encountered in image segmentation of tracing the boundary of an object. Tracking contours as a function of time has been addressed in the pioneer work of Kass [11], in relation to localizing features in an image. Here we add more complexity to the situation in [11] since the motion of the agents starts far away from the boundary. Moreover, once the boundary is located the contour of agents moves along that boundary rather than keeping a fixed location.

The idea of adapting image processing techniques to simulate motions of swarms was proposed in [2], where the image segmentation technique of locating a contour with a model known as a snake [11], or energy-minimizing curve, [12] is adopted. We extent that analogy by allowing the formation of agents to move on a time-dependent surface or on a surface whose boundary travels in time. In the current work we explore the problem of simulating the motion of agents which effectively locate and follow the boundary of a surface of interest, assuming that the boundary is known to each agent and it is moving in time possibly along with the entire surface. The motion of agents takes place in either 2D or 3D.

The idea of the algorithm we present is to model the group of agents as a contour that deforms towards the boundary of the object. Interactions between nearby agents are assumed, the modeling of these interactions being analogous to having forces acting 
between nearby particles as in an elastic band. An energy functional results from this physical model in a continuum limit. The desired motion of the agents is obtained by minimizing this functional to which an appropriate goal dynamics term is added, so that its minimum is obtained as the agents land on the proposed target.

The algorithm assumes that each agent has the position of the boundary, has information on nearby agents, and evaluates its position on the surface as well as the local gradient of that surface. The movement of each agent can be modeled by a partial differential equation for the velocity of each vehicle. By discretizing this equation we derive a numerical scheme for the motion of each agent. Each equation contains a term accounting for movement along the steepest descent on the surface and a term corresponding to movement parallel to the tangent to the boundary that is being tracked. Coordinated behavior is created by imposing local interaction rules among agents. These terms also dictate the manner in which the formation will converge towards the desired goal.

The paper is organized as follows: In the next section we present an algorithm for an individual agent to accomplish the desired task. Then we continue in section III with adding a sparsing term which connects the motions of nearby swarmers and prevents collisions of agents in real applications. Obtaining collective motion local communication rules is essential. Typically these rules determine a general pattern of significance in applications [3]. Consequently in section III we present a PDE model that includes interaction between nearby swarmers. These interactions are based on modeling the swarm as the discretization of an elastic string with elastic and bending forces acting between nearby particles. In section IV we give a numerical method based on the model in the previous section. We continue in section $\mathrm{V}$ with the corresponding algorithm in $3 \mathrm{D}$, which means the motion now takes place on a surface defined in $3 \mathrm{D}$ rather then $2 \mathrm{D}$. We end with a conclusion section. 


\section{The Collective Motion Algorithm}

The simplest version of the algorithm consists in designing motion terms that would lead a single vehicle to move towards a given boundary and then move along that boundary. The component of the motion that determines the approach to a prescribed boundary consists in moving along the steepest descent direction along a surface. To make the vehicle describe the boundary we include a term that is parallel to the tangent to the boundary of the surface. The velocity of a vehicle is thus calculated from these two components giving a system of two ordinary differential equations.

Let $C(x, y)$ denote the concentration function of the environment at the agent's position $(x, y)$. By "concentration", we mean a function which describes any spatially varying variable, such as density, temperature, etc. Let $P(x, y)=f(C(x, y))=\left(C-C_{0}\right)^{2}$ be a function that achieves a minimum at the boundary of the environmental concentration. Let $v=v(x, y)$ denote the position

vector. The motion according to the rule $\frac{d v}{d t}=-\nabla P$ results in a gradient descent toward a local minimum of the function $P$, therefore towards the curve $C=C_{0}$. Also the rule $\frac{d v}{d t}=\nabla^{\perp} C$, (where $\nabla^{\perp} C$ denotes the normal vector to the boundary of the surface) results in travel along the boundary of the concentration surface $C$. Combining the two terms mentioned above we arrive at the following model:

$$
\begin{aligned}
& \frac{d x}{d t}=-\partial_{x} P-\omega \frac{\partial_{y} C}{\|\nabla C\|} \\
& \frac{d y}{d t}=-\partial_{y} P+\omega \frac{\partial_{x} C}{\|\nabla C\|}
\end{aligned}
$$

The parameter $\omega$ in Eq. 1 determines the speed of the vehicle in the direction of the tangent to the boundary. Following [2] as a test case, we consider as the concentration function: 


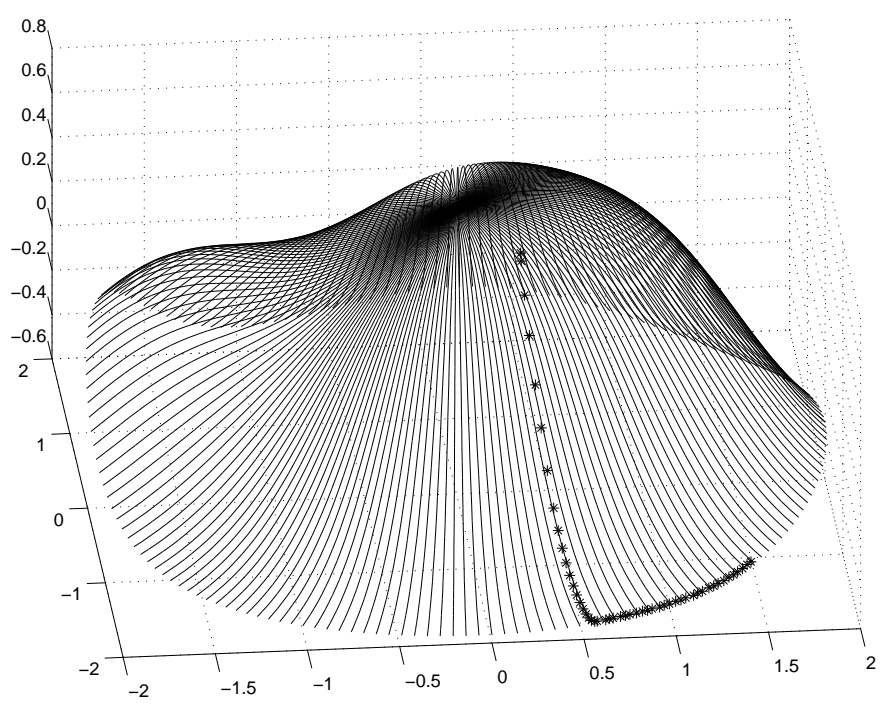

Fig. 1. The trajectory of a single agent (represented by '*'s) launched on a concentration surface, at initial condition $x 0=0.3$ and $y 0=0.5$, reaching the boundary and accurately following the boundary. Solid lines denote the surface $C(x, y)$.

$$
\begin{aligned}
& C(x, y)=\tanh \left(F(x, y)-\frac{3}{4}\right) \\
& F(x, y)=\sum_{i=1}^{4} \exp \left(\frac{-\left(\left(x-x_{i}\right)^{2}+\left(y-y_{i}\right)^{2}\right)}{\sigma^{2}}\right) .
\end{aligned}
$$

Equation 2 is defined on a disk of radius 2 and $\left(x_{i}, y_{i}\right)=(1,0)$, $\left(0,-\frac{1}{2}\right),\left(-\frac{3}{2}, \frac{1}{2}\right),\left(\frac{3}{20}, 1\right)$ for $i=1,2,3,4$ and $\sigma=1.0 . C_{0}$ is the concentration on the boundary, so it is obtained from Eq. (2 ) taking $x$ and $y$ on the boundary of the circle of radius 2.0 centered at the origin. In this way we are targeting a boundary that varies in space.

We solve the Eq. ( 1 ) subject to Eq. 2 by using Runge-Kutta method in time to obtain the trajectory shown in Fig. 1, where the initial conditions were $x_{0}=0.3$ and $y_{0}=0.5$. We also show a top-down view of the same trajectory in Fig. 2 where the level set of the surface are shown as well as the boundary of the surface (the circle of radius two) on which the agent moves.

To achieve movement along the boundary we calculate $C_{0}$ using Eq. ( 2 ) for $x$ and $y$ corresponding to coordinates on the circle 


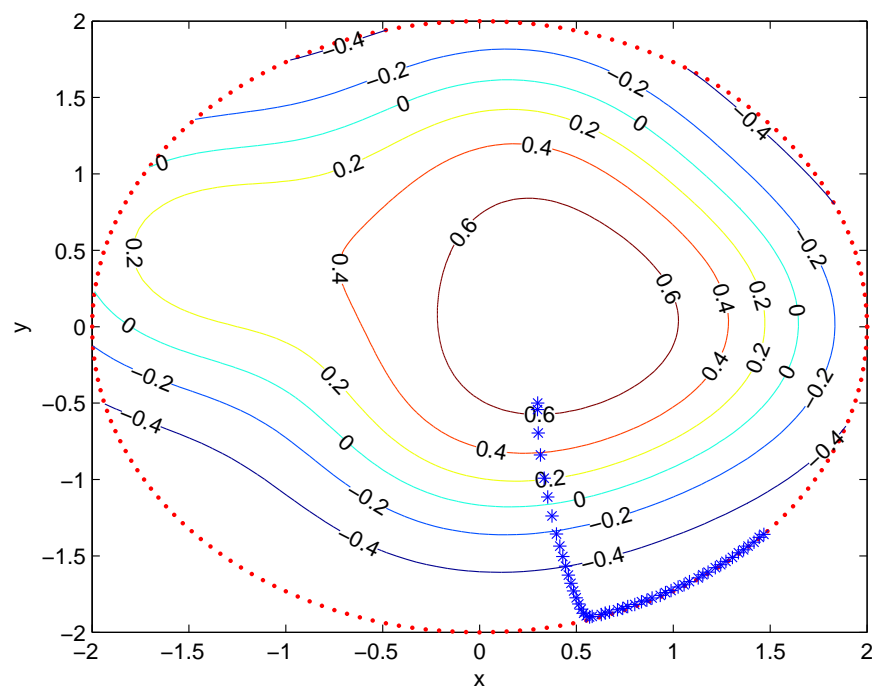

Fig. 2. Top-down view of the surface and trajectory in Fig.1. Level sets of the projected surface are shown. The boundary of the surface is projected onto the circle of radius 2 .

of radius 2 where the surface is defined (any other curve in $x-y$ space could have been considered).

The algorithm can be further extended to allow a vehicle to target a boundary which moves in time. In this case, Eq. (1) is used again, the only difference being that $C_{0}$ is now a function that varies in time as well in space. In Fig. 3 we show three nearby trajectories following a boundary which moves in time at a constant speed $v_{0}=2$. Four different instances in time are shown at $t=0.6,1,1.5$ and $t=2.0$.

The existence and uniqueness theorem for ordinary differential equations [13] guarantees that the trajectories of this motion will not intersect. In realistic situations when agents are used, collision among agents can become a concern. For such a situation we add a sparsing term in Eq. 11meant to repel nearby vehicles [14]. This term varies the velocity of a vehicle according to the following equations:

$$
\frac{d v_{i}}{d t}=\sum_{v_{j_{j \neq i}}} \nabla U\left(v_{j}, v_{i}\right), \quad v_{i}=\left(x_{i}, y_{i}\right)
$$



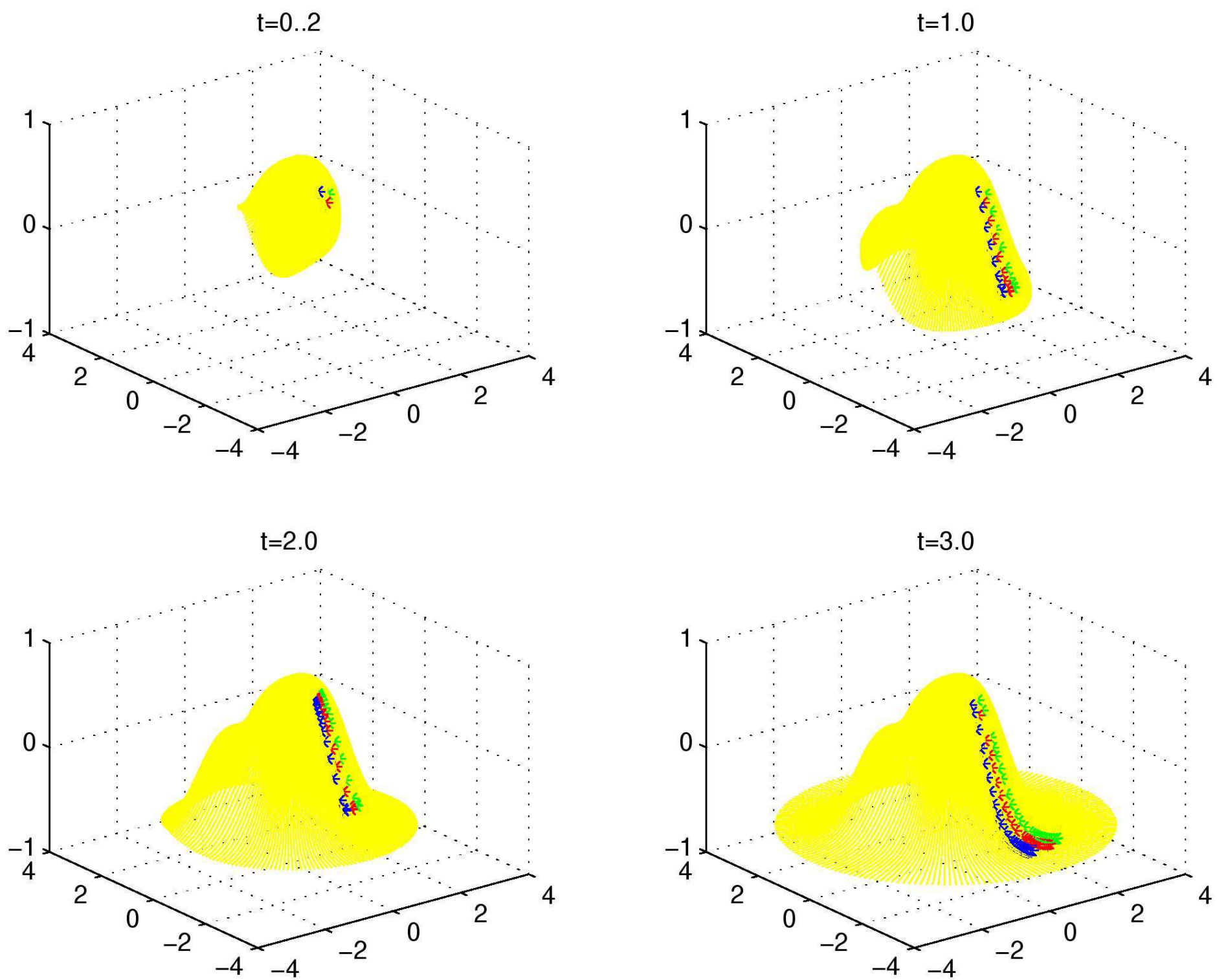

Fig. 3. Three nearby trajectories evolving on a concentration surface whose boundary expands in time at a constant speed. The four figures show the trajectories at $t=0.2,1.0,2.0,3.0$. Solid lines denote the evolving concentration surface.

where the repulsion kernel is given by $U(v, w)=C_{r} \exp \left(-\frac{|v-w|}{l_{r}}\right)$. Here the parameter $l_{r}$ is a length scale. In what follows $C_{r}=10$, and $l_{r}=0.5$. This term is set non-zero only when the distance between vehicles is less than a given cut-off distance $R$; i.e., a maximum distance at which the sparsing is active. In Fig. 4b) we show 4 trajectories whose motion is given by the Eq. 1 to which the sparsing term in Eq. 3 has been added. For comparison the same trajectories without the sparsing term are shown in Fig. 

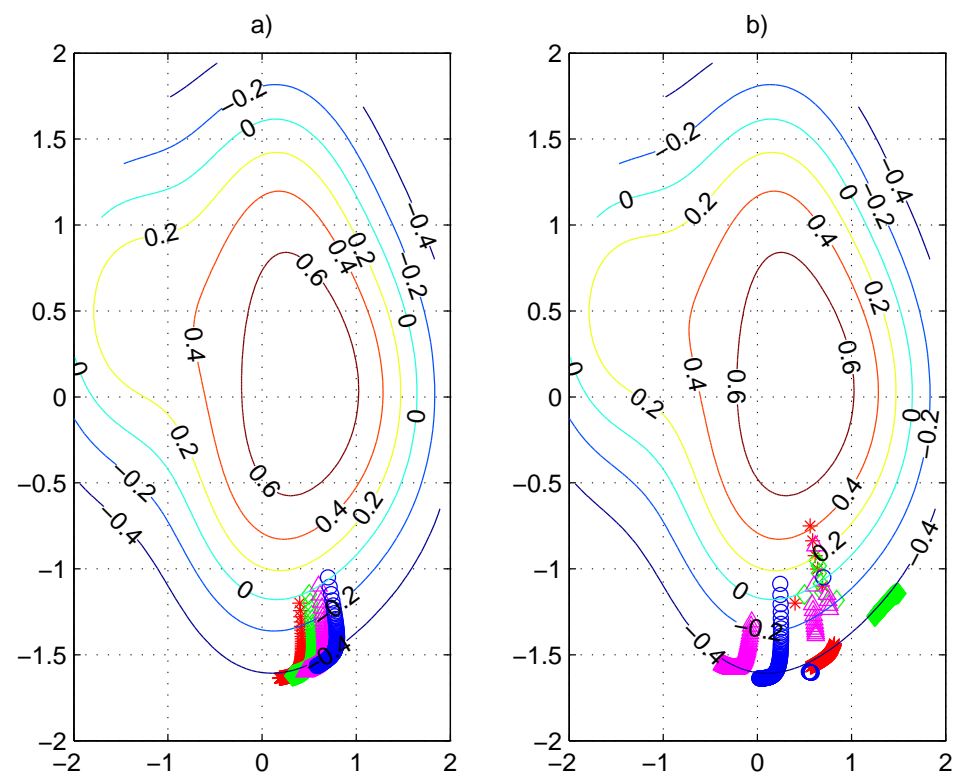

Fig. 4. Four trajectories, on the concentration surface starting with the same initial conditions. (a) There is no communication between agents. The trajectories evolve in time independently. (b)Similar to (a) but with a sparsing term added into the equations of motion, so that agents that get too close repel each other.

4a). When sparsing is added, we see the 4 trajectories, shown in different colors, intermingle several times on the surface until smooth trajectories are established. This differs from the orderly trajectories that can be seen in Fig. 3.

The above algorithm is used when only a few independent vehicles are available. When a sufficient number of vehicles are available, direct interaction among nearby vehicles can be introduced. This accounts for communication among vehicles in real situations and for creating desirable patterns of the motion of a swarm, since a swarm motion is essentially defined as creating various overall patterns and behaviors out of local interaction rules. In what follows the approach will be to view the group of agents as a continuous curve moving and expanding in time. A nice continuum mechanical analogy occurs when the motion is being thought of as an elastic band that expands and bends aiming to fit around a given boundary [11]. This approach will be shown in the next section. 


\section{Modeling the swarm behavior as an energy minimizing curve}

The algorithm we develop is meant to be used by vehicles with sensing capabilities moving through a medium of variable concentration and aimed at detecting a certain object such as the boundary of a surface. Interaction between vehicles can be conceived in various ways leading to a variety of behaviors of the overall swarm. In what follows we adopt the point of view of considering the group of agents modeled as an elastic object with elastic and bending forces acting between nearby agents [11, [1]. Achieving the desired goal amounts to solving a minimization problem. We summarize the related theory of this approach in this section and give the corresponding numerical discretization in the next section along with numerical examples.

The collective motion is modeled as the motion of a deformable contour which expands and wraps around the boundary, then moves around the boundary. Consequently it will be represented by a mapping:

$$
\Omega=S^{1} \rightarrow R^{2}, s \mapsto v(s)=(x(s), y(s))
$$

where $S^{1}$ is the periodic unit interval. Periodic boundary conditions are assumed since any agents in the swarm are considered to be identical. The algorithm consists in minimizing a certain functional $E$ over a space of admissible deformations $F$. This functional $E: F \rightarrow R$ represents the energy of the contour and has the following form:

$$
E(v)=\int_{\Omega}\left[w_{1}\left|v_{s}(s)\right|^{2}+w_{2}\left|v_{s s}(s)\right|^{2}+P(v(s))\right] d s,
$$

where the subscripts denote differentiation with respect to the Lagrangian parameter s which is the arc length, and $\mathrm{P}$ is a function of the environmental data. $\mathrm{P}$ also is designed to incorporate the goal dynamics we are interested in; i.e. the boundary of a surface. The other two terms of the functional $E$ account for the 
mechanical properties of the contour of agents; i.e., elasticity and bending. The first term makes the contour have stretch and the second term makes the contour bend [11]. The choice of the parameters $w_{1}$ and $w_{2}$ determine the elasticity and the rigidity of the contour.

If $v$ is a local extremum of $E$ it satisfies the associated EulerLagrange equation:

$$
\partial_{s}\left(w_{1} v_{s}\right)-\partial_{s s}\left(w_{2} v_{s s}\right)+\nabla P=0
$$

with periodic boundary conditions. As before let $P=\left(C-C_{0}\right)^{2}$. The relationship between potential functions and desired goals is discussed comprehensively in [12] and [11].

Since the collective motion we are designing takes place in time, we approximate the solution of Eq. (4) as the steady state of the following partial differential equation for the contour $v$ :

$$
\partial_{t} v=\partial_{s}\left(w_{1} v_{s}\right)-\partial_{s s}\left(w_{2} v_{s s}\right)+\nabla P
$$

in which the right hand side is the Euler-Lagrange Eq. 4. This equation describes how each point on the active contour $v$ should move in order to minimize the functional $E$. In the next section we show how this equation can be discretized and used in numerical examples.

\section{Collective Motion Algorithm along a Virtual Contour}

In this section we present an algorithm for collective motion based on the minimization approach presented above. This algorithm is obtained discretizing Eq. (51) and considering each spatial discretization point as representing an agent. Thus a group of agents will evolve according to a time-dependent scheme and will move along a virtual contour which is an approximation of the curve $v$ solving Eq. ( 5). 
We rewrite Eq. ( 5) as follows:

$$
\begin{aligned}
& \partial_{t} x=\alpha x_{s s}-\beta x_{s s s s}+\partial_{x} P \\
& \partial_{t} y=\alpha y_{s s}-\beta y_{s s s s}+\partial_{y} P
\end{aligned}
$$

where as previously $P(x, y)=\left|\left(C(x, y)-C_{0}\right)\right|^{2}$. Using finitedifferencing to discretize the derivatives with respect to the Lagrangian parameter $s$, we obtain:

$$
\begin{aligned}
\partial_{t} x_{i}=\frac{\alpha}{h^{2}}\left(x_{i+1}-2 x_{i}+x_{i-1}\right)- & \frac{\beta}{h^{4}}\left(x_{i-2}-4 x_{i-1}+6 x_{i}-4 x_{i+1}+x_{i+2}\right) \\
& +\partial_{x} P\left(x_{i}, y_{i}\right) \\
\partial_{t} y_{i}=\frac{\alpha}{h^{2}}\left(y_{i+1}-2 y_{i}+y_{i-1}\right)- & \frac{\beta}{h^{4}}\left(y_{i-2}-4 y_{i-1}+6 y_{i}-4 y_{i+1}+y_{i+2}\right) \\
& +\partial_{y} P\left(x_{i}, y_{i}\right)
\end{aligned}
$$

This discretization gives the equation of motion for a group of agents occupying the positions $\left(x_{i}, y_{i}\right)$. We define thus the motion of the mobile agents by a set of coupled ODEs for the positions of the $N$ agents. In what follows we take $\alpha=0.01$ and $\beta=0.0001$ as in [2].

The equations of motion given by ( 7 ) will evolve agents towards the boundary of the concentration surface. Once on the boundary, movement along the boundary is generated by adding a term parallel to the normal to the surface as in (1). This results in the following scheme: 


$$
\begin{gathered}
\partial_{t} x_{i}=\frac{\alpha}{h^{2}}\left(x_{i+1}-2 x_{i}+x_{i-1}\right)-\frac{\beta}{h^{4}}\left(x_{i-2}-4 x_{i-1}+6 x_{i}-4 x_{i+1}+x_{i+2}\right) \\
+\partial_{x} P\left(x_{i}, y_{i}\right)-\omega \frac{\partial_{y} C\left(x_{i}, y_{i}\right)}{\left.\mid \nabla C\left(x_{i}, y_{i}\right)\right) \mid}+\sum_{j \neq i} \partial_{x} U\left(\left(x_{i}, y_{i}\right),\left(x_{j}, y_{j}\right)\right) \\
\partial_{t} y_{i}=\frac{\alpha}{h^{2}}\left(y_{i+1}-2 y_{i}+y_{i-1}\right)-\frac{\beta}{h^{4}}\left(y_{i-2}-4 y_{i-1}+6 y_{i}-4 y_{i+1}+y_{i+2}\right) \\
+\partial_{y} P\left(x_{i}, y_{i}\right)+\omega \frac{\partial_{x} C\left(x_{i}, y_{i}\right)}{\left.\mid \nabla C\left(x_{i}, y_{i}\right)\right) \mid}+\sum_{j \neq i} \partial_{y} U\left(\left(x_{i}, y_{i}\right),\left(x_{j}, y_{j}\right)\right) .
\end{gathered}
$$

The last term represents the sparsing term that has been added as in Eq. 3. In the examples below we let $\omega=2.0$ and the time derivative in Eq. 6] has been discretized using the forward Euler method. With this time discretization it follows that one and respectively two-nearest neighbor communication will occur, as seen from the discretization of the diffusion term and the discretization of the 4th order derivative. Stability issues as well an implicit version concerning this algorithm have been discussed in [1] where it is shown that stability of the forward Euler scheme requires the condition $\Delta t<\frac{h^{4}}{8 \beta+2 \alpha h^{2}}$, where $h$ stands for the distance between nearby agents.

Using Eq. ( 8) one can effectively target and track a level curve of a concentration surface. We have allowed in Eq. (8) the concentration on the boundary to be a function of $x$ and $y$. In Fig. 5. we show a swarm of 25 vehicles at four different instances in time, each one 20 time steps apart with a time step $\Delta t=0.01$. We show the swarm at the beginning of the motion which starts in the center of the surface, then we show three later pictures of the same swarm as it circles the surface boundary. Fig. 6] is a top-down view of Fig. 5 in which the projection of the surface boundary, (i.e. the circle of radius two) is marked so that one can clearly see the movement of the agents along this boundary. Figure [6] shows a top-down view of Fig. 5] in which the projected boundary of the surface (that is the circle of radius two) is marked and this enables one to see the accuracy of the motion 
of the swarmers on this boundary.

In Fig. 7 we pick one of the vehicles shown in Fig. 5 and show the relative error evolution for this particular trajectory. The chosen trajectory is the one that starts at $x_{0}=0.4 \cos \left(\frac{3 \pi}{4}\right)$, $y_{0}=0.4 \sin \left(\frac{3 \pi}{4}\right)$, and it shows a relative error which is below $2 \%$. The rest of the vehicles behave similarly.

Developing the algorithm further, in Eq. ( 8), we allow both the concentration $C$ and the boundary $C_{0}$ to be functions of time as well as space. To simulate variation in time we consider the same surface as before given by (2) and vary both $x$ and $y$ periodically in time with different frequencies according to:

$$
x=A_{0} \cos \left(\frac{2 \pi t}{40}\right) \quad y=A_{0} \sin \left(\frac{2 \pi t}{30}\right) .
$$

This results in a generic, time-dependent change of the whole surface. Here we show in Fig. 8 four images of the motion at various moments in time. In Fig. 9 we show the relative error associated with the motion of one of the trajectories in Fig. 8 , We chose again the same initial conditions as for Fig. 7. This error averages out to $3.6 \%$ when $A_{0}=0.1$ and stays below $5 \%$. If we raise $A_{0}=0.15$, the corresponding average error will be $5.3 \%$.

\section{Collective motion in $3 \mathrm{D}$}

The above algorithm can be realized in 3D by a similar variational formulation. Initially the minimization problem was introduced in image processing [15] as a deformable surface model meant to evolve to a certain location and shape. We extend that idea to the motion of a collective of agents. We consider the group of agents placed on a deformable surface which evolves in time to fit a certain boundary surface. The deformation assumes elastic and bending forces that are internal to the deformable surface. The forces have to balance the so-called external forces that are stretching this surface so that it will fit a boundary surface or 

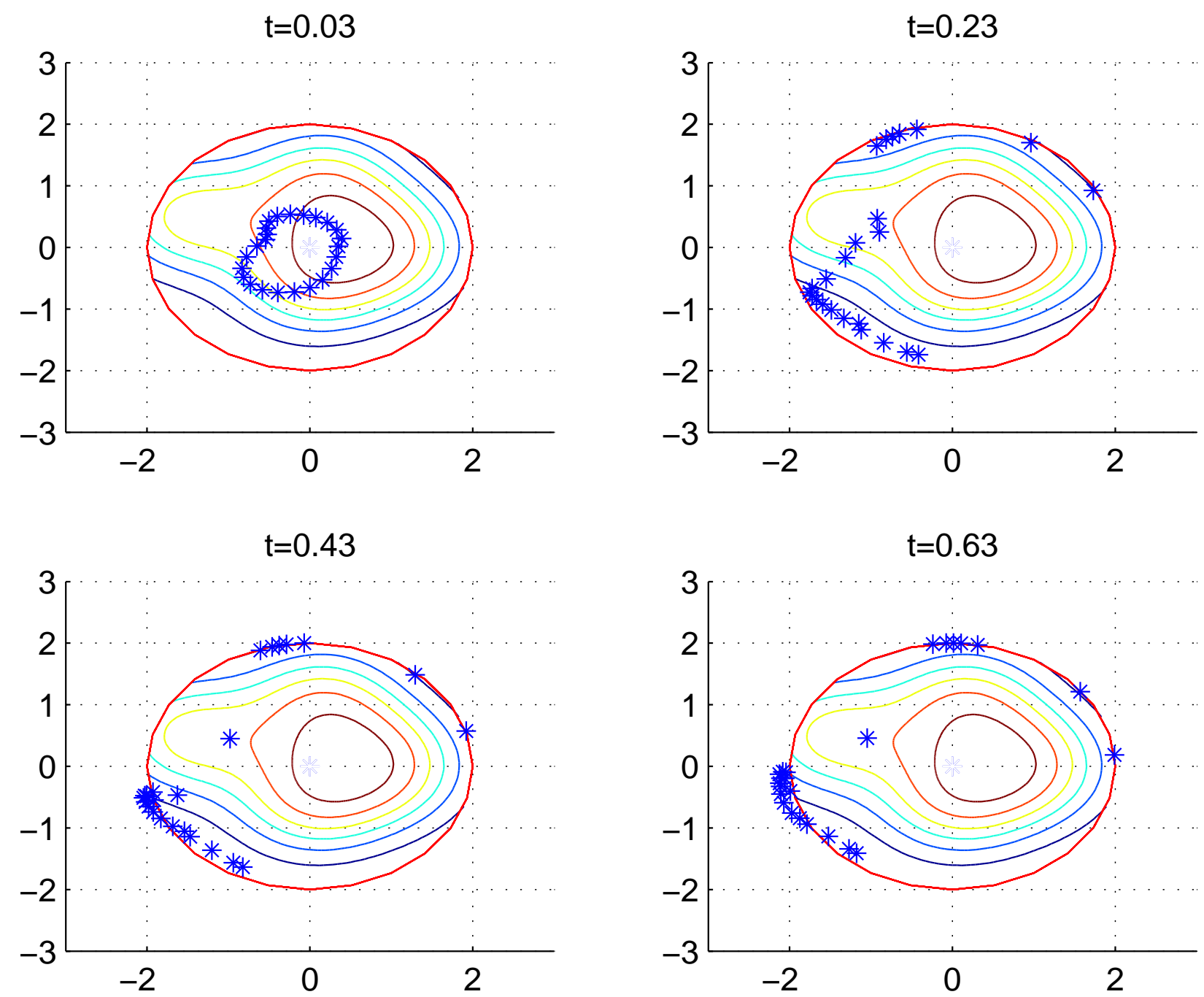

Fig. 6. The picture gives a top-down view of the swarm in Fig.5. The algorithm accurately follows a boundary variable in space which, when projected forms a circle of radius 2 .

$$
\begin{aligned}
\Omega & =[0,1] \times[0,1] \rightarrow R^{3} \\
v(s, r) & =(x(s, r), y(s, r), z(s, r)) .
\end{aligned}
$$

The associated energy $E$ is given by: 


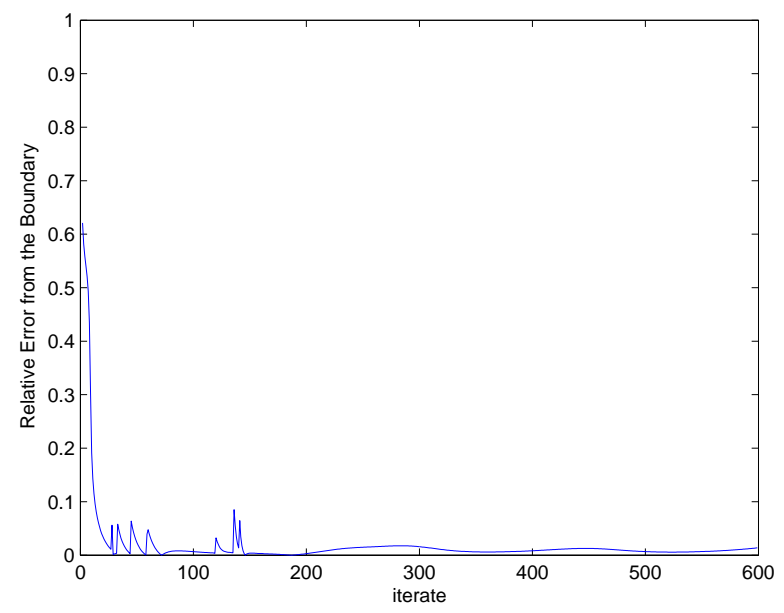

Fig. 7. The picture shows the relative error corresponding to one of the trajectories in Fig. 5. which is seen to stay below $2 \%$

$$
\begin{aligned}
E(v)= & \int_{\Omega}\left[w_{10}\left|v_{s}(s)\right|^{2}+w_{01}\left|v_{r}(s)\right|^{2}+2 w_{11}\left|v_{s r}(s)\right|^{2}\right. \\
& \left.+w_{20}\left|v_{s s}(s)\right|^{2}+w_{02}\left|v_{r r}(s)\right|^{2}+P(v(s, r))\right] d s d r
\end{aligned}
$$

where $P$ is associated to the task to be achieved by the algorithm. The boundary is now a $2 \mathrm{D}$ surface in space. Similar to the $2 \mathrm{D}$ case we let $P(x, y, z)=\left(C(x, y, z)-C_{0}\right)^{2}$ where now $C$ and $C_{0}$ are functions of $x, y$ and $z$. The other terms in Eq. 10 determine how the motion in space of the surface $v$ will take place. Continuing our analogy from continuum mechanics, these terms model internal forces that are acting on the shape of the surface $v$ as it moves towards its goal. Consequently the shape of the surface $v$ will depend on the elasticity coefficients $w_{10}, w_{01}$, the rigidity coefficients $w_{20}, w_{02}$ and the resistance to twist $w_{11}$. We also constrain the surface $v$ by assuming periodic boundary conditions.

A local minimum $v$ of $E$ satisfies the associated Euler-Lagrange equation:

$$
\begin{gathered}
-w_{10} v_{s s}(s, r)-w_{01} v_{r r}(s, r)+2 w_{11} v_{s s r r}(s, r)+w_{20} v_{s s s s}(s, r) \\
+w_{02} v_{r r r r}(s, r)=-\nabla P(v(s, r))
\end{gathered}
$$

to which boundary conditions are added. Equation [1] represents 

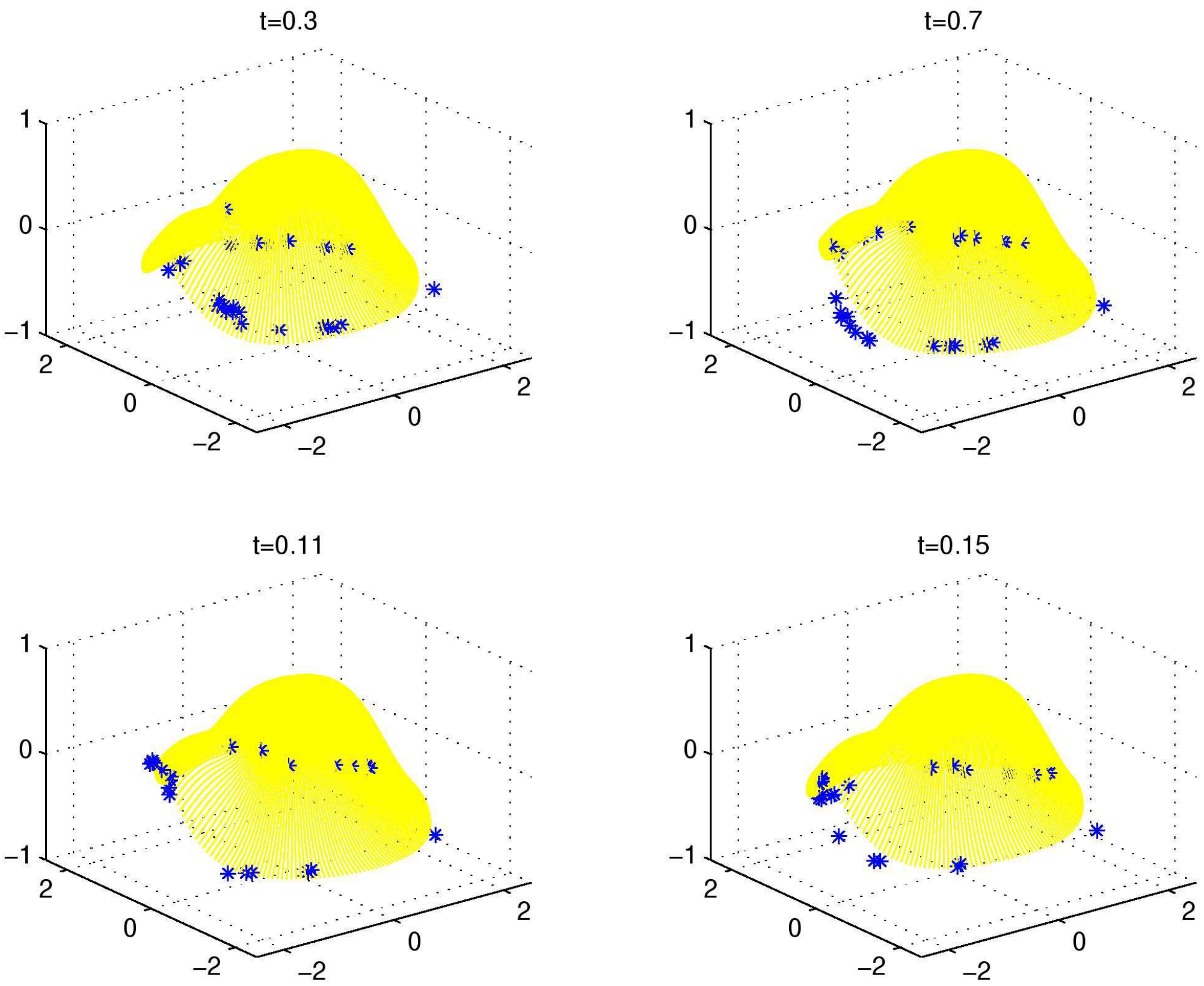

Fig. 8. The picture shows four instances in time of the motion of a swarm of vehicles on a time-varying surface. The motion is shown at $t=0.3,0.7,0.11,0.15$, the time step was $\Delta t=0.01$

the necessary condition for a minimum of $E,\left(E^{\prime}(v)=0\right)$. A solution of Eq. 11 can be seen as either realizing the equilibrium between internal and external forces or as reaching a minimum of the energy $E$. Since there may be many local minima, appropriate initial data $v_{0}(s, r)$, leading to the desired minimum is assumed when solving the associated evolution equation, in which we add 


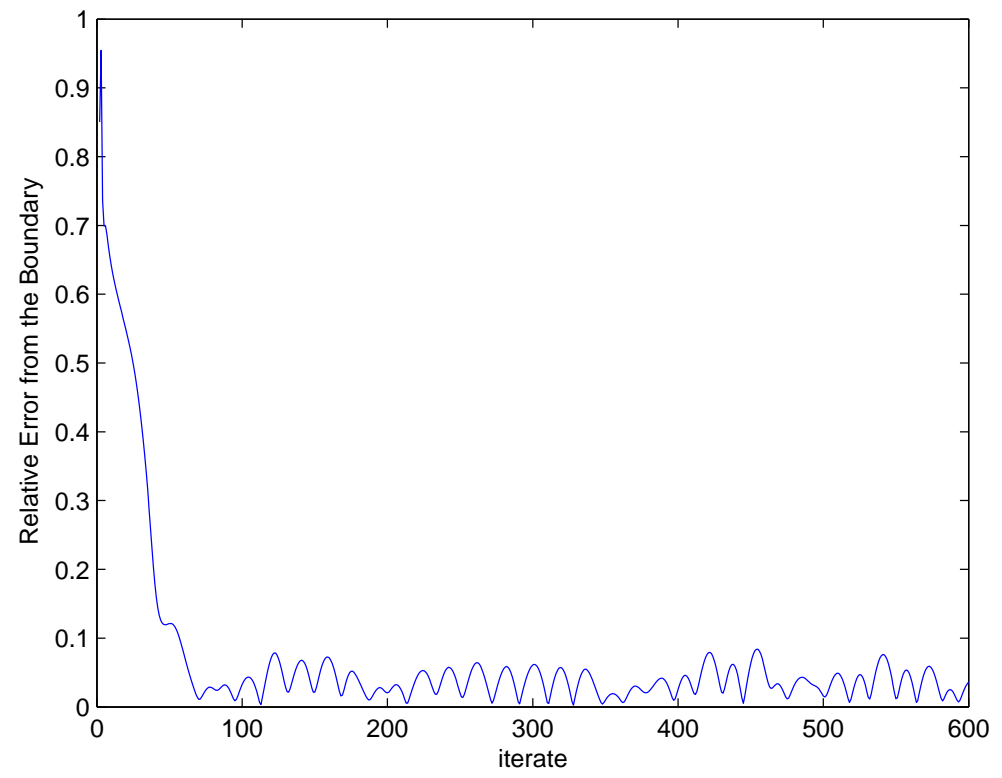

Fig. 9. The relative error in the motion of a single vehicle chosen at random out of the 25 trajectories in Fig. 8 The error averages 3.6\%.

a temporal parameter $t$ :

$$
\begin{gathered}
\frac{\partial v}{\partial t}-w_{10} v_{s s}(s)-w_{01} v_{r r}(s)+2 w_{11} v_{s s r r}(s)+w_{20} v_{s s s s}(s) \\
+w_{02} v_{r r r r}(s)=-\nabla P(v(s, r)) .
\end{gathered}
$$

A solution to the static minimization problem is obtained when the solution $v$ converges as $t$ tends to infinity, which means that the term $\frac{\partial v}{\partial t}$ vanishes leading to a solution of the static problem.

The evolution Eq. ( 12) will be used to model the motion of a swarm in 3D. We discretize this equation in space using finitedifferences and each discrete spatial point will stand for the position of a vehicle proceeding analogously to the $2 \mathrm{D}$ case in the previous section. Thus this swarm will be designed as lying on the surface $v(s, r)$ and moving towards the assigned boundary with interactions between nearby vehicles determined by the elasticity and rigidity coefficients in Eq. (12). We apply this discretization (not presented here, but standard and analogous to the discretization in section IV) to the motion of a specific swarm evolving on a concentration volume defined in 3D of equation: 


$$
\begin{aligned}
& C(x, y, z)=\tanh \left(F(x, y, z)-\frac{3}{4}\right) \\
& F(x, y, z)=\sum_{i=1}^{4} \exp \left(\frac{-\left(\left(x-x_{i}\right)^{2}+\left(y-y_{i}\right)^{2}+\left(z-z_{i}\right)^{2}\right)}{\sigma^{2}}\right)
\end{aligned}
$$

where $\left(x_{i}, y_{i}\right)=(1,0,-1),\left(0,-\frac{1}{2}, \frac{1}{2}\right),\left(-\frac{3}{2}, \frac{1}{2}, 0\right),\left(\frac{3}{20}, 1,-\frac{1}{2}\right)$ for $i=$ $1,2,3,4$ and $\sigma=1.0$.

The swarm will move in space through a medium of variable concentration in space. The swarm will move along the steepest descent direction which is given now by a 3 -dimensional vector $\left(\frac{\partial P}{\partial x}, \frac{\partial P}{\partial y}, \frac{\partial P}{\partial z}\right)$.

A last numerical example shows that the motion of the swarm in 3D can be directed to reach and track a specific curve on the boundary. We define a curve in space lying on the spherical boundary. This curve is defined by the equation:

$$
\begin{aligned}
& x=\cos (\phi) \cos (\phi) \\
& y=\cos (\phi) \sin (\phi) \\
& z=\sin (\phi)
\end{aligned}
$$

When using the discretization of Eq. [1] with this modified goal dynamics we obtain the 14 trajectories seen in Fig. 10. The goal dynamics is introduced in the discretization of Eq. 11 by evaluating $C_{0}$ at $x, y$ and $z$ given by 14 . In Fig. 10 we show both the exact target curve (in red)a and the curve described by 14 swarmers (in blue) as they approximate movement along the curve of Eq. 14. In this figure we remark that the target curve is traveled by the swarmers in opposite directions. We also show a detail of Fig. 10 in Fig. 11 where arrows indicate how the trajectories evolve from inside the sphere towards the surface boundary and then move along the prescribed curve.

As in the $2 \mathrm{D}$ case we now vary the concentration in time. In the 
concentration equation, Eq. 13, we let $\mathrm{x}, \mathrm{y}$ and $\mathrm{z}$ vary in time according to the formula:

$$
x=A_{0} \cos \left(\frac{2 \pi t}{40}\right) \quad y=A_{0} \cos \left(\frac{2 \pi t}{30}\right) \quad z=A_{0} \cos \left(\frac{2 \pi t}{20}\right)
$$

The corresponding figure is not shown here since it is very similar to Fig. 10 with the exception that the sphere undergoes slight deformations in time due to the motions given in 15. To illustrate the time-dependent case we give in Fig. 12 the relative error for one agent. Namely the one starting at: $x_{0}=0.8 \cos \left(\frac{4 \pi}{5}\right) \cos \left(8 \frac{\pi}{25}\right)$, $y_{0}=0.8 \cos \left(\frac{4 \pi}{5}\right) \sin \left(8 \frac{\pi}{25}\right), z_{0}=0.8 \sin \left(\frac{4 \pi}{5}\right)$. It can be seen in this figure that the relative error is below $5 \%$.

\section{Conclusions}

We have presented an algorithm that allows a formation of agents to move towards a prescribed boundary and then describe that boundary. The motion takes place on stationary or non-stationary surfaces and can be realized as dynamics on a surface (2D) or as motion in space (3D).

The algorithm represents a new development over snake algorithms used in image processing [11], [2]. Those algorithms were primarily designed to detect level-sets in an image. Here we show that a similar algorithm will detect boundaries that vary in space and in time. Our new algorithm, along with the one in [2], directs the idea of snake algorithms towards solving a new problem, namely the problem of controlling motions of swarms. Another development was to allow the environment in which the swarm is moving to depend on time in a quite general manner.

In future work we will extend the current algorithm to detecting curves with a more complex topology than the one considered here. Our main goal in future developments of these algorithms 


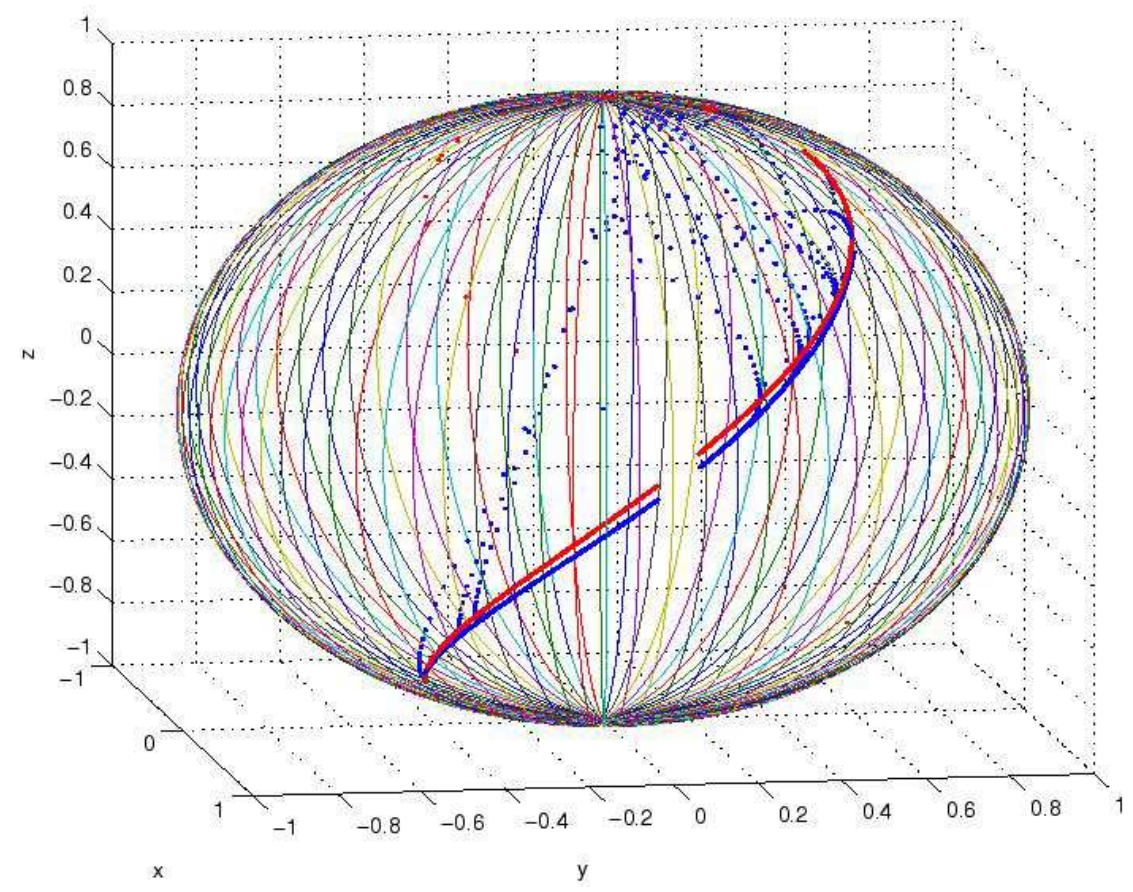

Fig. 10. 14 trajectories starting inside the spherical domain, reaching the boundary and following a specified curve on the boundary.

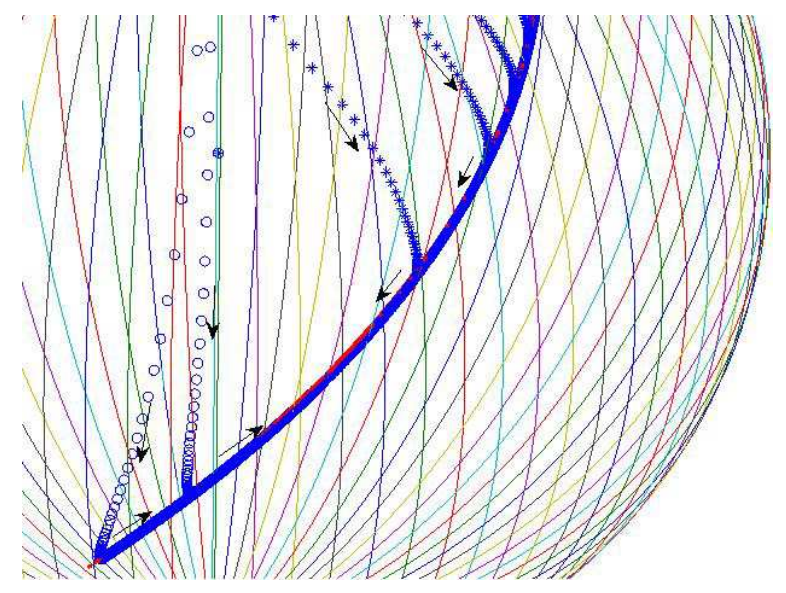

Fig. 11. Blowup of Fig. 10, where the arrows show the direction of the agent's trajectories.

will be to couple the equations of motion of the swarm with meaningful dynamical systems with relevance for biological application, chemical reactions and advective flows. In this way the abstract goal of reaching a certain surface or feature will be directed towards real-life applications. 


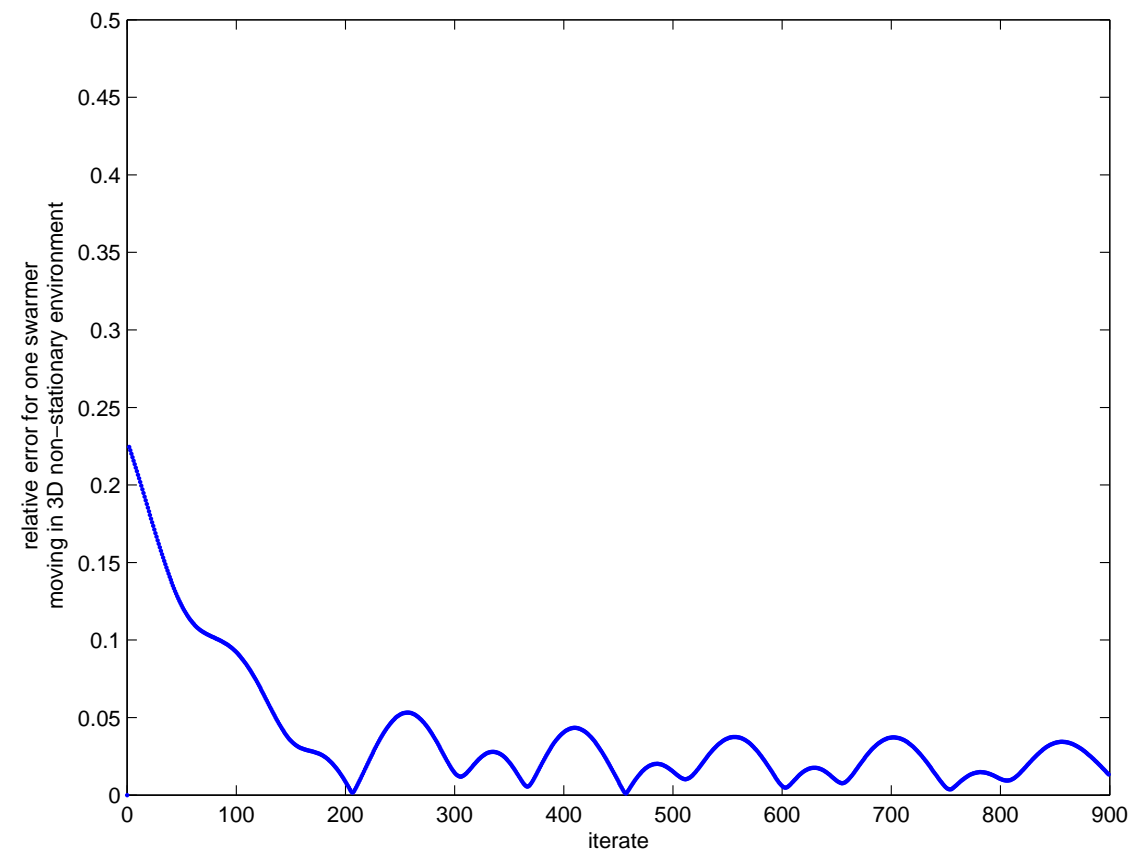

Fig. 12. The relative error in the motion of a single swarm reaching and then moving along a space and time-dependent boundary in 3D.

\section{Acknowledgment}

This research has been supported by the Office of Naval Research.

\section{References}

[1] Daniel Marthaler and Andrea L. Bertozzi, Collective motion algorithms for determining environmental boundaries, Autonomous Robots, special issue on Swarming, submitted 2002.

[2] A.L. Bertozzi M. Kemp, and D. Marthaler, Determining Environmental Boundaries: Asynchronous communication and physical scales, in Proceedings of the Block Island Workshop on Cooperative Control 2003, Lecture Notes in Control and Information Systems, edited by V. Kumar, N. Leonard, S. Morse (September 2003), Springer-Verlag.

[3] "Swarming: Network Enabled C4ISR, 13-14 Jan 2003. Conference Proceedings, http://search.netscape.com/ns/.

[4] Eric Bonabeau, Marco Dorigo and Guy Theraulaz, "Swarm Intelligence- From Natural to Artifical Systems", a volume in the Santa Fe Institute Studies in the Sciences of Complexity, Oxford University Press, 1999.

[5] J.K.Parrish and L. Edelstein-Keshet, "Complexity, Pattern and Evolutionary Trade-Offs in Animal Aggregation", Science Vol. 284, 5411, pages 99-101, 1999. 
[6] Riccardo Cassinis, "Landmines Detection Methods Using Swarms of Simple Robots", Intelligent Autonomous Systems 6, E.Pagello et al. (Eds) IOS Press, 2000 .

[7] A. Stentz and M. B. Dias, "A Free-Market Architecture for Coordinating Multiple Robots", CMU-RI-TR-99-42, The Robotics Institute, Carnegie Mellon University, Dec. 1999.

[8] W.Burgard, M.Moors, D.Fox, R. Simmons and S. Thrun, "Collaborative MultiRobot Exploration", Proceedings of IEEE International Conference on Robotics and Automation, April 2000.

[9] J.P.Dessai, I.Ostrowski and V.Kumar, "Controlling formations of multiple mobile robots", Proc. of IEEE International Conference on Robotics and Automation, May 1998.

[10] Petter Ogren, Edward Fiorelli and Naomi Ehrich Leonard, "Formations with a Mission: Stable Coordination of Vehicle Group Maneuvers", Proc. Symposium on Mathematical Theory of Networks and Systems, August 2002.

[11] Michael Kass, Andrew Witkin and Demetri Terzopoulos, "Snakes: Active Contour Models", International J. of Computer Vision, 321-331 (1988).

[12] Guillermo Sapiro, "Geometric Partial Differential Equations and Image Analysis", Cambridge University Press, 2001.

[13] Earl A. Coddington and Norman Levinson, "Theory of Ordinary Differential Equations", McGraw-Hill Book Company, 1955.

[14] A.Mogilner, L.Edelstein-Keshet, L.Bent, A. Spiros Mutual interactions, potentials, and individual distance in a social aggregation, to be published.

[15] Isaac Cohen, Laurent D. Cohen and Nicholas Ayache, "Using Deformable Surfaces to Segment 3D Images and Infer Differential Structures", CVGIP: Image Understanding Vol.56, No.2 September, pp. 242-263, 1992. 